\section{Solid-State Electrochemical Micromachining Enables Direct Surface Patterning}

Electrochemical micromachining (EM) has been used as a "shaving off" method to fabricate textured or patterned surfaces. It has become one of the most widely used methods because it requires simple equipment and enables more rapid etching than techniques such as ion-beam milling and laser abrasion. However, during the EM process, a liquid electrolyte, which can be difficult to handle, is required as a conducting medium between the two electrodes. Furthermore, high-resolution patterning processes require masking or the use of focused laser writing or focused ion-beam writing of patterns. Now, K. Kamada of Kyushu University, K. Izawa of Kumamoto University, and their colleagues have proposed an electrochemical micromachining procedure that eliminates the need for wet processing and allows fine patterns to be written.

As reported in the April 19 issue of Chemistry of Materials (p. 1930; DOI: $10.1021 / \mathrm{cm} 0502929)$, the researchers describe a route for solid-state micromachining using an anodic electrochemical reaction at the microcontact between an ion-conducting microelectrode and a metal substrate, which they call the solidstate electrochemical micromachining (SSEM) method (schematically shown in Figure 1). During the SSEM process, the metal substrate is locally incorporated into the ion-conducting microelectrode in the form of metal ions through the microcontact under a dc bias. Continuous application of an electric field produces micromachining of the substrate, thereby removing the need for wet processing.

The microelectrode is formed from a pyramid-like (tetrahedral shape) piece of $\beta^{\prime \prime}-\mathrm{Al}_{2} \mathrm{O}_{3}$ (typically, $\mathrm{Na}-\beta^{\prime \prime}-\mathrm{Al}_{2} \mathrm{O}_{3}$ ) with a contact radius of $\sim 10 \mu \mathrm{m}$. It is very light-

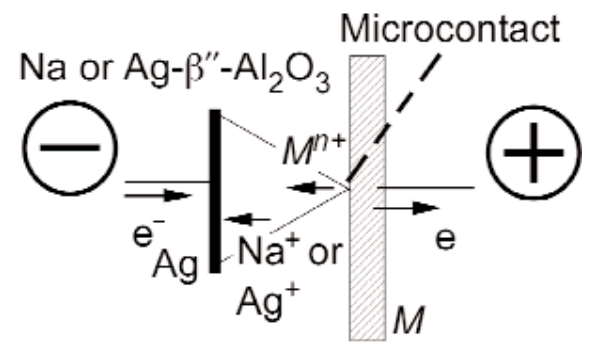

Figure 1. Model for ion migration during solid-state electrochemical micromachining of a metal substrate; $\mathrm{M}$ is the target metal substrate. Reprinted in part with permission from Chem. Mater. 17 (April 19, 2005) p. 1931. (02005 American Chemical Society. weight $(\sim 0.02 \mathrm{~g})$, which reduces the mechanical stress at the microcontact to a minimum and keeps it constant. The solid-state electrochemical cell consists of a $\mathrm{Ag}$ plate (cathode)/ pyramid-like $\mathrm{Na}-\beta^{\prime \prime}$ $\mathrm{Al}_{2} \mathrm{O}_{3}$ microelectrode/ target metal substrate (thickness, $0.5 \mathrm{~mm}$; M: Ag or $\mathrm{Zn}$; anode) system. The researchers said that since the contact radius at the Na- $\beta^{\prime \prime}$ $\mathrm{Al}_{2} \mathrm{O}_{3} / \mathrm{M}$ interface is on the order of 10 $\mu \mathrm{m}$, a position-selective dissolution occurs at the microcontact, which makes it an excellent tool to pattern the metal surface directly in either a dot or scan-line format. The researchers also said that further work is needed to solve some remaining problems with this technique, including a slow etching rate, low current efficiency, and roughness of the micromachined surface.

FENGTING Xu

\section{Rethinking the Hydrophobicity of Lotus Leaves}

The lotus leaf is a symbol of purity in many cultures due to its ability to remain clean. When water drops fall on the leaves, they exhibit a superhydrophobic contact angle of $160^{\circ}$; the drops promptly roll off the leaves, collecting any dirt along the way. This behavior is believed to be a result of the complex structure of the leaf, which includes a wax layer and a twolevel surface structure of micrometer-scale bumps and nanometer-scale hair-like features. In contrast to previous studies that observed the behavior of macroscopic water drops placed on the lotus leaf surface, Y.-T. Cheng from General Motors Research and Development Center and D. Rodak from Ricardo Meda Technical Services conducted experiments to investigate the hydrophobicity of this surface when water condenses on the leaves.

As reported in the April 4 issue of Applied Physics Letters (144101; DOI:10.1063/ 1.1895487), when water condensed on the leaf during exposure to water vapor, the researchers found three types of behavior: some drops would fall off the leaf as expected; some drops formed a high contact angle but would not roll off; and some others spread out, wetting the leaf. The researchers said the behavior arises primarily from the two-level structure. When macroscopic water drops are placed on the leaf in standard studies, they are in contact with the leaf bumps and the air trapped between the bumps and the hairlike structure. The resulting contact angle is a combination of the contact angle of the leaf and air.

However, during condensation, microscopic water drops can form in the nanometer-scale structure, interpenetrating the hair-like structure. When water drops are placed on the leaf surface following condensation treatment, the drop is supported by a composite surface formed by the leaf and water, decreasing the overall contact angle. In the last case, the surface may even be hydrophilic.

According to the researchers, this finding significantly changes the common perception of lotus leaves as superhydrophobic and has important ramifications on how to make and use superhydrophobic surfaces, with potential applications on self-cleaning and low-friction surfaces.

MARIA MARTA FIDALGO

\section{Zeolite-Coated Optical Fibers Demonstrated as Sensitive Chemical Vapor Detectors}

From manufacturing to pollution monitoring to homeland security, many applications await the development of fast and accurate chemical-vapor sensors. H. Xiao and a team of researchers from the New Mexico Institute of Mining and Technology have reported constructing just such a sensor based on a cleaved optical fiber coated with a thin zeolite film. The sensor is able to detect isopropanol and other test vapors in a nitrogen atmosphere at concentrations as low as $0.075 \%$. The technology is especially promising for use in the field because it has excellent thermal and chemical stability.

The use of optical-fiber technology in gas sensors is well established, particularly since optical measurements can be made with high precision. Zeolites-crystalline aluminosilicate materials with regular nanometer-scale pores-have also been used in chemical sensing applications, acting as molecular sieves. Until now, however, these technologies have not been combined. As reported in the June 1 issue of Optics Letters (p. 1270), the researchers first cleaved a $9 \mu \mathrm{m}$ (core diameter) singlemode optical fiber and grew a thin-film coating of all-silicon MFI-type zeolite on the exposed fiber face. They then placed the coated fiber end in a nitrogen atmosphere with a controlled flow of isopropanol and directed light at $1539.5 \mathrm{~nm}$ down the fiber toward the coated face. The refractive index and thus boundary reflectivity of zeolites depends strongly on the presence of guest molecules in the pores and channels. By measuring the intensity of reflected light from the zeolite coating, the researchers could determine the amount of isopropanol it had adsorbed from the atmosphere. The sensor showed a monotonic reflectivity response to low vapor concentrations and very fast adsorption, taking $1 \mathrm{~s}$ to adsorb introduced isopropanol and $3 \mathrm{~s}$ to return to the base reflectivity when the isopropanol flow was 
turned off. The researchers noted several possible improvements to the system, including optimization of the zeolite chemistry and the use of more sophisticated optical reflection measurement techniques.

COLIN MCCORMICK

\section{Highly Ordered Isoporous Membranes Fabricated from Nanocomposites}

Isoporous films, which have application in photonics, biotechnology, and biomedical devices, have previously been formed by the incorporation of inorganic nanoparticles into an organic polymer matrix. However, the properties of this class of nanocomposite materials are adversely affected by nanoparticle aggregation. In general, attachment of polymer chains to nanoparticles increases their dispersion and results in improved mechanical properties and thermal stability. Recently, D. Nystrom and colleagues from KTH, together with $\mathrm{M}$. Whittaker from the University of Queensland, grafted polymers onto silica nanoparticles and used them to fabricate isoporous membranes.

As reported in Macromolecular Rapid Communications 26 (p. 524; DOI: 10.1002/ marc.200400617), KTH researcher A. Hult and colleagues used atom-transfer radical polymerization to graft polystyrene (PS) onto functionalized silica nanoparticles. Infrared spectroscopy confirmed the PS grafts, which had a molecular weight of $23,400 \mathrm{~g} / \mathrm{mol}$. The researchers cast films on a glass substrate from a mixture of hybrid nanoparticles, $\mathrm{CS}_{2}$, and linear PS under humid conditions. A temperature decrease resulting from solvent evaporation caused water to condense onto the film surface. Stabilized water droplets were then formed when hybrid nanoparticles precipitated at the water-solvent interface. An opaque film formed when all solvent evaporated, with the final membrane containing $10 \mathrm{wt} \%$ silica. The researchers, using an optical microscope and atomic force microscopy to analyze the membrane structure, observed a hexagonal array of pores, that is, a honeycomb structure, with an average pore diameter of $2.5 \mu \mathrm{m}$, a nearly monodisperse pore size distribution, and a pore depth varying from $0.8-1.5 \mu \mathrm{m}$. By changing the humidity and the rate of air flow, the researchers were able to vary the pore size from $2 \mu \mathrm{m}$ to $8 \mu \mathrm{m}$.

Hult and co-researchers said that their method is applicable to a broad range of materials and that the large surface areas, open pore structure, and isoporous nature of the films make them very attractive for membrane applications.

Hult said, "We can also make nano- composites in which copolymers are grafted to the silicon microstructure."

STEVEN TROHALAKI

\section{Ice Used As Resist for Patterning Nanostructures}

G.M. King and researchers at Harvard University have recently demonstrated the use of frozen water as a resist for electronbeam lithography. They patterned chromium lines as narrow as $17 \mathrm{~nm}$ and lines of local surface chemical transformations as narrow as $5 \mathrm{~nm}$, as reported in the June 8 issue of NanoLetters (p. 1157; DOI: 10.1021/nl050405n).

The researchers cooled a silicon substrate in a scanning electron microscope chamber to $128 \mathrm{~K}$ before leaking in water vapor to grow a stable ice film. They patterned the ice film using the electron beam (1-30 keV, 30-150 pA, 5 nm diameter) and performed a lift-off process by sput-

\section{CNT Arrays Encapsulated into Freely Suspended Flexible Films}

Carbon nanotubes (CNTs), with their mechanical strength and electrical properties, are excellent candidates for multifunctional membrane sensors requiring high electrical conductivity and extreme robustness. However, it is hard to obtain longliving, freestanding, organized micro- and nanostructured membranes composed of nanowires and nanotubes due to their extreme fragility. Recently, $\mathrm{H}$. Ko and a group of researchers at Iowa State University introduced a method to fabricate freestanding microarrays of CNTs by encapsulating them into robust, albeit compliant, polymeric nanofilms.

As reported in the May 17 issue of Chemistry of Materials (p. 2490; DOI: $10.1021 / \mathrm{cm} 050495 \mathrm{x})$, the researchers encapsulated CNT microarrays into freely suspended layer-by-layer (LbL) membranes by using spin-assisted LbL (SA-LbL) assembly and microcontact printing by sacrificial polymer patterning. The experimental procedure for patterned arrays of CNTs is outlined schematically in Figure 1. The CNT layer was $\sim 5 \mathrm{~nm}$ thick with a density of $\sim 18$ bundles $/ \mu^{2}{ }^{2}$. Raman spectra at the CNT area confirmed that neither the oxidation process nor deposition routine significantly affected the microstructure of the CNTs encapsulated into the LbL membranes. High optical contrast caused by the alternating layers into the LbL membrane creates an efficient Raman grating with a variation of G-band intensity of 1:1000 and higher.

By using interferometry, the researchers also studied how the encapsulation of the CNT arrays affects elastic properties of the freely suspended films. The result confirms that the filler-toughening mechanism effectively enhances the elastic
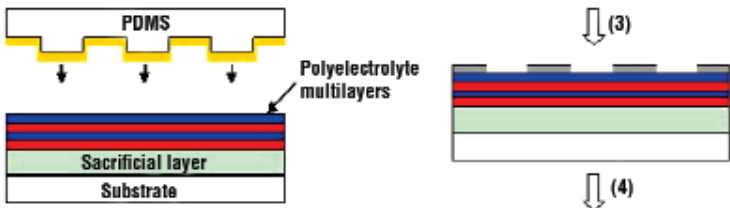

П (4)

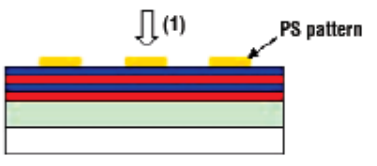

$\sqrt{\text { (2) }}$
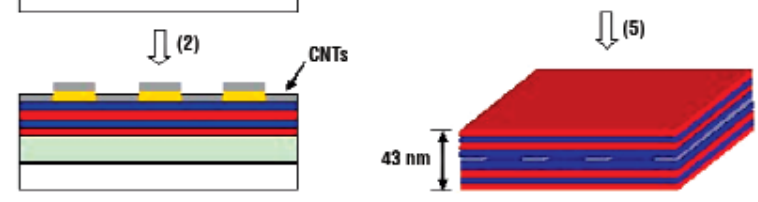

Figure 1. Schematic illustration of the procedure for fabricating freely suspended carbon nanotube arrays: (1) microcontact printing of polystyrene (PS) onto polyelectrolyte multilayers; (2) deposition of carbon nanotubes on the patterned substrates; (3) removal of PS layers; (4) formation of topmost polyelectrolyte multilayers by layer-by-layer assembly; and (5) release of carbon nanotube arrays by rinsing away the supporting sacrificial film. PDMS is poly(dimethylsiloxane). Reprinted in part with permission from Chem. Mater. 17 (May 17, 2005) p. 2490. ${ }^{\circ} 2005$ American Chemical Society. properties of the patterned nanomembranes similarly to that demonstrated for thick homogeneous LbL films.

The researchers said that the excellent mechanical properties of CNTs can be retained and finely tuned by embedding freely suspended carbon nanotube arrays in nanoscale polymer films. New anisotropic properties such as optical (Raman) gratings and potentially directional conductivity can be introduced. These new anisotropic properties have prospective applications for directional sensing and anisotropic electrical conduction.

TAO XU 